\title{
Photodissociation of Small Group-11 Metal Cluster Ions: Fragmentation Pathways and Photoabsorption Cross Sections
}

\author{
M. Vogel and A. Herlert \\ Institut für Physik, Johannes-Gutenberg-Universität, D-55099 Mainz, Germany \\ L. Schweikhard \\ Institut für Physik, Ernst-Moritz-Arndt-Universität, D-17487 Greifswald, Germany
}

\begin{abstract}
Noble metal cluster ions $C u_{n}^{+}, A g_{n}^{+}$and $A u_{n}^{+}(\mathrm{n}=3-21)$ have been stored in a Penning trap and photodissociated by low intensity laser pulses of $10 \mathrm{~ns}$ at photon energies of $3.49 \mathrm{eV}$ and 4.66 $\mathrm{eV}$. The fragmentation pathways, neutral monomer and dimer evaporation, have been monitored as a function of cluster size, excitation energy and element. It is found that the behavior of the branching ratio between monomer and dimer evaporation as a function of excitation energy depends on the metal under investigation. In particular, the slope of the energy dependence is positive for silver but negative for gold and copper cluster ions. Furthermore, photoabsorption cross sections are determined from observed total fragment yields in single-photon dissociation. (J Am Soc Mass Spectrom 2003, 14, 614-621) (c) 2003 American Society for Mass Spectrometry
\end{abstract}

$\mathrm{T}$ The dissociation pathway of an excited polyatomic system gives valuable information on structural and dynamical properties. For most clusters of group-11 metals (copper, silver and gold), neutral monomer evaporation is the only relevant decay pathway. For some cluster sizes, however, neutral dimer evaporation is known to be a strong competitor [1-13].

This competition can be described in terms of the statistical nature of the unimolecular dissociation process. The decay pathway branching ratio is a function of the cluster size $n$, the excitation energy $E$ and the respective dissociation barriers $D_{1}(n)$ for monomer evaporation and $D_{2}(n)$ for dimer evaporation $[14,15]$. For cluster cations of the noble metals the decay pathway branching ratio $f_{2} / f_{1}$ in the unimolecular dissociation process

$$
\begin{array}{cl}
M_{n}^{ \pm} & \nearrow M_{n-2}^{ \pm}+M_{2} \\
& \searrow M_{n-1}^{ \pm}+M
\end{array}
$$

depends on the excitation energy [14] with relative fragment yields $f_{2}$ for neutral dimer evaporation and $f_{1}$ for neutral monomer evaporation. Due to the larger corresponding phase space, evaporation of neutral dimers from small excited clusters is preferred. However, in most cases it is depressed by a higher dissociation energy $D_{2}(n)>D_{1}(n)$. In the present work a quantitative description of the decay pathway compe-

Published online May 2, 2003

Address reprint requests to Manuel Vogel, Institut für Physik, Universität Mainz, 55099 Mainz, Germany. E-mail: Manuel.Vogel@Uni-Mainz.De tition as a function of element, cluster size and excitation energy is given.

In addition, the total fragment yields have been measured at well-defined excitation parameters, which allows a determination of photoabsorption cross sections. It is well known that the optical properties of finite systems as small as the metal clusters under investigation deviate significantly from metal bulk behavior. While the optical absorption spectrum of the bulk has few characteristic features [16, 17], mediumsize systems tend to have narrower absorption regions, as has been shown in measurements on gold and silver particles on the nm-scale [18]. Small systems with high symmetry, such as the closed-shell systems $A u_{9}^{+}$and $A u_{21}^{+}$have a relatively sharp absorption resonance with a width on the order of $0.5 \mathrm{eV}$ [19]. In general, small systems show more features due to geometry-related and electronic effects [17].

Even with experiments at fixed wavelengths such as the present one, a pronounced size-dependence of the optical absorption behavior is observed. In contrast to earlier measurements the photoexcitation parameters are set at fixed values. Thus the observed yields allow a direct comparison with respect to element $(\mathrm{Cu}, \mathrm{Ag}, \mathrm{Au})$, cluster size $(n=3-21)$ and photon energy $(3.49 \mathrm{eV}, 4.66 \mathrm{eV})$.

\section{Experimental Setup and Procedure}

The details of the setup (Figure 1) have already been given elsewhere [20-22]. The experimental sequence consists of the following steps: 


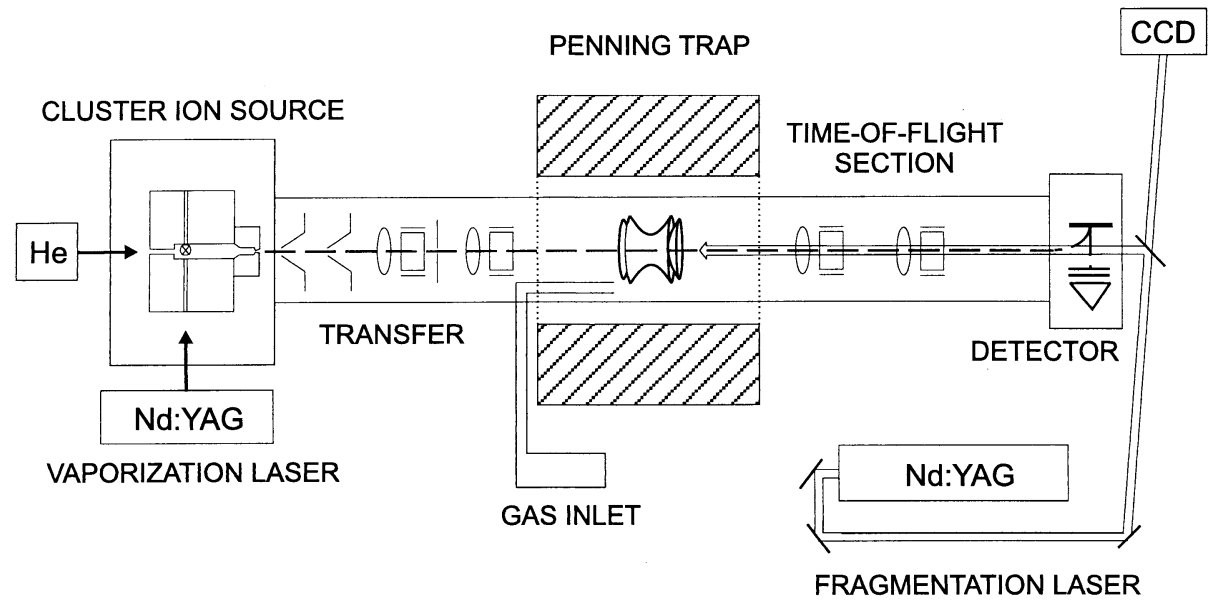

Figure 1. Overview of the experimental setup.

- Cluster ion production in a Smalley-type laser vaporization source [23, 24].

- Transfer to the Penning trap [25] where the clusters are captured in flight [26] and stored.

- Selection of the cluster size of interest by resonant radial ejection of all other clusters.

- Radial centering of the size-selected cluster ion ensemble in the trap by use of inert gas and quadrupolar excitation of the ion motion (axialization [27, 28]).

- Photoexcitation by a 10-ns pulse of a frequencymultiplied Nd:YAG laser.

- Storage period of $100 \mathrm{~ms}$.

- Analysis of the resulting cluster ion ensemble by ejection from the trap into a time-of-flight (TOF) mass spectrometer with single-ion counting [20, 21].

The pulses from a Nd:YAG laser are frequencytripled or frequency-quadrupled by use of non-linear BBO crystals to produce wavelengths of $355 \mathrm{~nm}$ or 266 $\mathrm{nm}$, respectively. A beam splitter (Suprasil) is used to deflect $5 \%$ of the resulting laser intensity into a CCD camera which allows for an on-line observation of the laser beam position and of the geometrical properties of the laser pulse, i.e., the radial profile and the diameter. In the following, the laser beam diameter is defined by the 2- $\sigma$-width of a Gaussian fit to the observed radial laser intensity profile. The laser pulse energy for all present measurements was kept constant at a value of $(30 \pm 2) \mu \mathrm{J}$ with a laser beam diameter of $d_{L}=(2.0 \pm$ $0.2) \mathrm{mm}$

Figure 2 shows the essential part of the experimental sequence in terms of TOF spectra. Frame A gives the trapped ion distribution as transferred from the cluster ion source. The center of this distribution can be shifted by varying the relative electric potential of cluster source and ion trap [20]. The remaining ion signal after mass selection (here: $A u_{13}^{+}$) is shown in Frame B. Frame $\mathrm{C}$ shows the abundance spectrum after laser excitation of the selected $A u_{13}^{+}$ensemble. In this case, the resulting relative dimer fragment yield $f_{2}$, i.e., the dimer fragment vs. total fragment yield, is about $75 \%$.
In general, cluster dissociation occurs delayed and can be monitored time-resolved [6, 29-31]. For the present experiments the decay time window has been set to the comparatively long time of $100 \mathrm{~ms}$. In principle, for a certain combination of cluster size, stability and excitation energy it is possible that the time scale of the fragmentation process is of the order of this experimental time window or larger. This would result in the observation of a smaller fragment yield than the true value and thus in an underestimation of the photoabsorption cross section. However, for systems as small as the present ones the dependence of the fragmentation time scale on the excitation energy is very strong. Typically, an increase in excitation energy of $10 \%$ re-

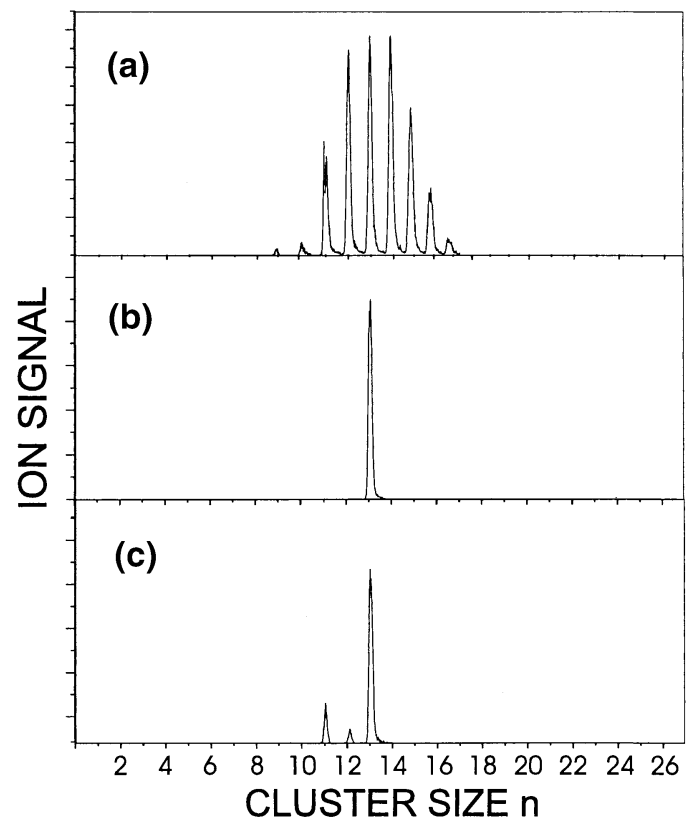

Figure 2. Steps in the experimental sequence in terms of timeof-flight mass spectra: (a) Trapped ion distribution as transferred from the trap. (b) Remaining ions after mass selection (here: of $\left.A u_{13}^{+}\right)$. (c) Mass spectrum after laser excitation of the $A u_{13}^{+}$ensemble with a $266 \mathrm{~nm}$ laser pulse of $30 \mu \mathrm{J}$ pulse energy. 
sults in an increase of the fragmentation rate by more than one order of magnitude. Therefore, it can be assumed that in all cases where fragmentation is observed, it occurs completely within the given observation time window.

In order to improve the statistical significance of the measurements, several hundred experimental cycles with some tens of ions each are performed and combined in one abundance spectrum. Furthermore, the measurement cycles are alternated with reference cycles that are identical with the exception of the application of the laser pulse. Thus, the effect of the photoexcitation can clearly be identified. The photoexcitation energy is identical to the energy that leads to dissociation of the cluster if no radiative energy loss occurs before decay. Extrapolation from bulk properties as well as comparison with the well-discussed case of vanadium clusters [32] suggests that radiation can be ruled out for the present experiment.

\section{Results and Discussion}

\section{Decay Pathway Branching Ratios}

Figure 3 shows the observed relative $M_{n-2}^{+}$fragment yield $f_{2}$ as a function of cluster size for clusters $C u_{n}^{+}, A g_{n}^{+}$ and $A u_{n}^{+}(\mathrm{n}=3-21)$.

Clusters consisting of an even number of atoms $n$ show exclusive monomer evaporation irrespective of their size. In the case of clusters with odd n, for all three elements under investigation there is a characteristic transition from dimer evaporation for small cluster sizes to monomer evaporation for large cluster sizes. This transition region ranges from size $n=7$ to $n=13$ for copper, $\mathrm{n}=9$ to $\mathrm{n}=13$ for silver and $\mathrm{n}=7$ to $\mathrm{n}=$ 15 for gold. For all three elements, the dimer fragment yield (i.e., the $M_{n-2}^{+}$fragment yield) for the 9-atomic ion $\left(\mathrm{M}_{9}^{+}\right.$with 8 atomic valence electrons) is smaller than that of both odd-numbered neighbors with $n=7$ and $\mathrm{n}=11$. This effect is particularly strong in the case of silver, intermediate for copper and comparatively weak for gold. Qualitatively, the effect can be related to the exceptional stability of the 9-atomic ion due to the electronic shell closing at 8 atomic valence electrons [33, 34, 13].

Note, that when the laser pulse energy is kept sufficiently low (as for the present studies), it has no influence on the ratio between monomer and dimer fragments. For an explicit confirmation, the laser pulse energy was varied between $30 \mu \mathrm{J}$ and $120 \mu \mathrm{J}$. As an example, the irradiation of $A u_{13}^{+}$at $\lambda=266 \mathrm{~nm}$ is shown in Figure 4.

This behavior confirms the absence of sequential decays, which in general lead to a more complicated functional behavior. From previous measurements [6, $13,14,29,31,35]$ it is known that for most of the cluster ions under study, a single photon of $\lambda=355 \mathrm{~nm}$ resp. $\lambda=266 \mathrm{~nm}$ wavelength is sufficient to induce a decay on the time scale of the experiment. Exceptions are the

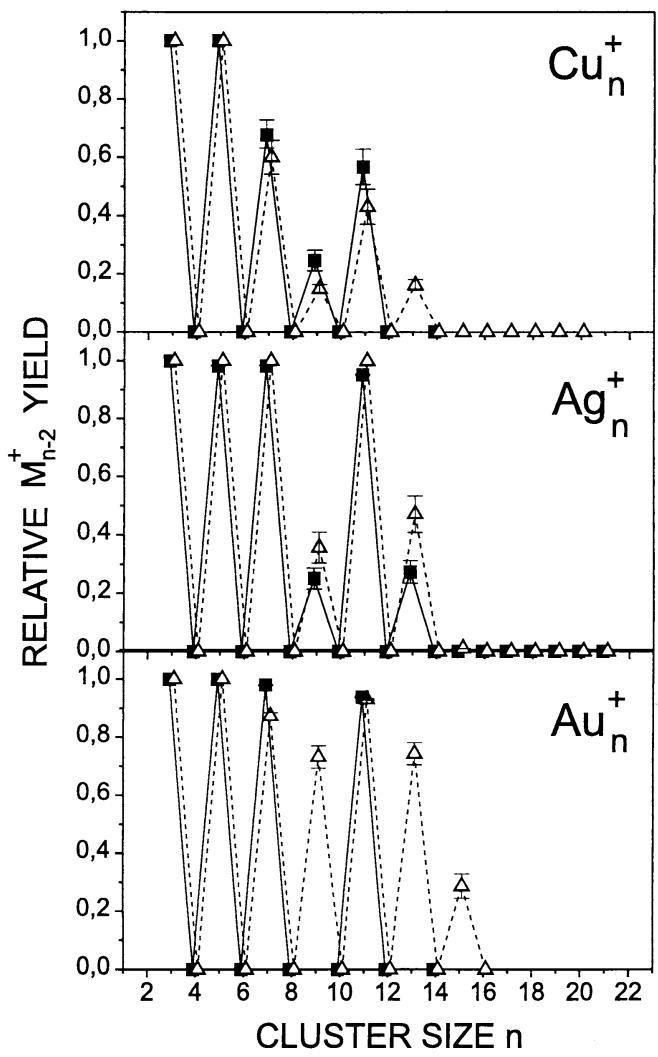

Figure 3. Observed relative $M_{n-2}^{+}$fragment yield as a function of cluster size for clusters $\mathrm{Cu}_{n}^{+}, A g_{n}^{+}$and $A u_{n}^{+}(\mathrm{n}=3-21)$. Data points for $\lambda=266 \mathrm{~nm}$ (open triangles) are connected by a dashed line. Full squares represent measurements at $\lambda=355 \mathrm{~nm}$. The two data sets are slightly shifted for better visibility. Missing data points indicate that no information on the relative $M_{n-2}^{+}$fragment yield can be given since no decay is observed at the respective photon energies (see also Figure 6).

very stable closed-shell systems $M_{9}^{+}$and $M_{21}^{+}$and large gold and copper clusters with $\mathrm{n}>16$ when irradiated with $\lambda=355$ nm-pulse (Figure 6). In these cases, a fragment yield equal to zero is explained by the excitation energy not exceeding the dissociation energies of

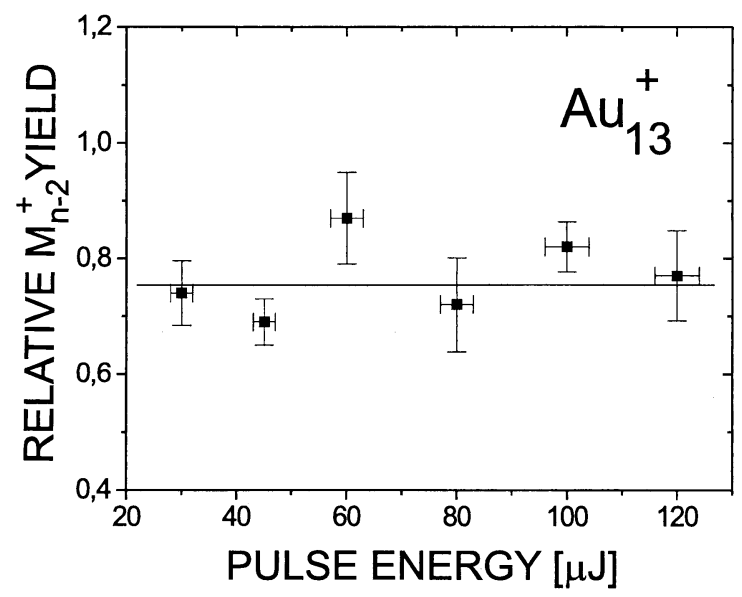

Figure 4. Relative $A u_{n-2}^{+}$yield of $A u_{13}^{+}$as a function of laser pulse energy at a wavelength of $\lambda=266 \mathrm{~nm}$. 


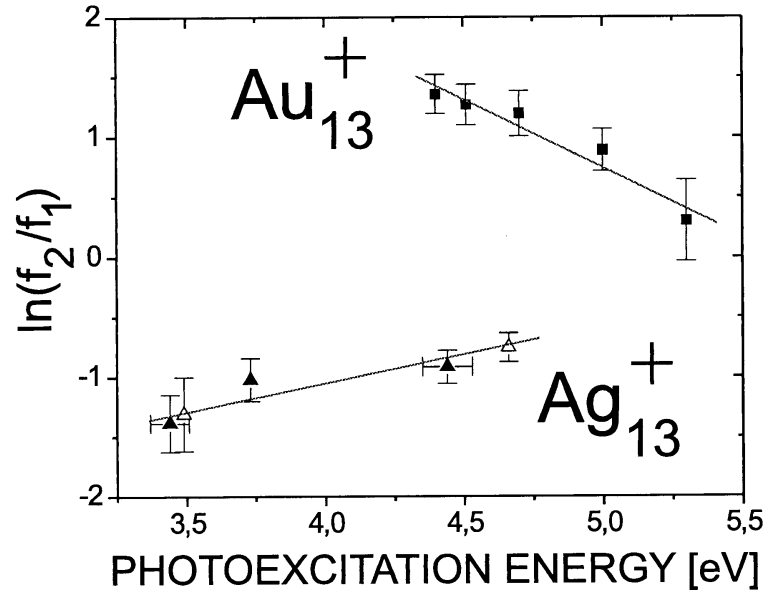

Figure 5. Logarithm of the observed decay pathway branching ratio $f_{2} / f_{1}$ as afunction of photoexcitation energy for $A u_{13}^{+}$(from [14]) and for $A g_{13}^{+}$(open triangles: this work, solid triangles: from [6]).

the respective clusters. However, single-photon absorption is in all cases insufficient to induce sequential decays. From $[6,13,14,29,31,35]$ it can be deduced that absorption of more than one photon of the present energies would in most cases induce sequential decay, which is not observed (see e.g., Figure 4). Additionally, in none of the present cases any significant signal of cluster sizes smaller than $\mathrm{n}-2$ was detected.

In contrast to the absence of any dependence from the laser pulse energy there is obvious influence of the photon energy on the ratio between monomer and dimer fragments. In Figure 5 the logarithm of the decay pathway branching ratio $f_{2} / f_{1}$ is plotted as a function of photoexcitation energy for $A u_{13}^{+}$(from [14]) and for $\mathrm{Ag}_{13}^{+}$. The corresponding error bars in energy shown in Figure 5 result from the uncertainty in dissociation energy and kinetic energy release of the sequential decay of $\mathrm{Ag}_{14}^{+}$.

The data points except for $\mathrm{E}=3.49 \mathrm{eV}$ and $\mathrm{E}=4.66$ $\mathrm{eV}$ are taken from [6] or result from two-step sequential dissociations of $A g_{14}^{+}$after two-photon absorption when the known dissociation energy $\mathrm{D}_{14}=1.83(6) \mathrm{eV}$ and the kinetic energy release of $A g_{14}^{+}$is subtracted from the respective two-photon excitation energy of the initial $A g_{14}^{+}$[6].

Most important, the slope of the energy-dependence has different signs for silver clusters as compared to gold and copper clusters. The sign is negative for all gold cluster ions with observable competition between dimer and monomer evaporation $(\mathrm{n}=7,9,11,13$ and 15) [14]. The same qualitative behavior is found for $\mathrm{Cu}_{7}^{+}$, $\mathrm{Cu}_{9}^{+}$and $\mathrm{Cu} u_{11}^{+}$. In contrast, the sign is positive for $\mathrm{Ag}_{13}^{+}$ and for the other silver cluster ions $\left(\mathrm{Ag}_{9}^{+}\right.$and $\left.A g_{11}^{+}\right)$that have been investigated in this respect (see also Figure 3). The slopes are given in Table 1.

From decay rate theory in the framework of the Weisskopf model $[15,36,37]$, the slope $s_{n}$ of the energydependence is given by [14]
Table 1. Slopes $s_{n}$ of the energy dependence of the fragmentation pathway braching ratio of clusters $\mathrm{Cu} u_{n}^{+}, \mathrm{Ag}_{n}^{+}$and $A u_{n}^{+}$. The numbers for gold are taken from [14].

\begin{tabular}{lccc}
\hline $\begin{array}{l}\text { Cluster } \\
\text { size } n\end{array}$ & $C u_{n}^{+}$ & $A g_{n}^{+}$ & $A u_{n}^{+}$. \\
\hline \hline 7 & $-0.32 \pm 0.08$ & - & $-0.93 \pm 0.09$ \\
9 & $-0.40 \pm 0.12$ & $+0.20 \pm 0.07$ & $-0.98 \pm 0.08$ \\
11 & $-0.48 \pm 0.09$ & $+0.31 \pm 0.12$ & $-1.13 \pm 0.13$ \\
13 & - & $+0.54 \pm 0.07$ & $-0.75 \pm 0.12$ \\
15 & - & - & $-0.59 \pm 0.15$ \\
\hline \multicolumn{5}{c}{$s_{n}=\frac{d}{d E} \ln \left(\frac{f_{2}}{f_{1}}\right)$} \\
\end{tabular}

where $T$ is the microcanonical temperature [15] of the respective product system, and $C$ is its heat capacity. $\mathrm{E}$ is the excitation energy and $D_{1}$ and $D_{2}$ are the monomer and dimer dissociation energies of the precursor. Thus, the sign of $s_{n}$ is determined by the relation between the dissociation barriers for monomer and dimer evaporation $D_{1}$ and $D_{2}$. For all gold clusters investigated $(\mathrm{n}=7$, $9,11,13$ and 15), the slope $s_{n}$ has experimentally been found to be on the order of $-1 \mathrm{eV}^{-1}$ [14]. This restricts $D_{2}$ not to exceed $D_{1}$ by more than about $10 \%$. Experimentally, however, it has been found in model-independent measurements $[31,38]$ that in the relevant size region $D_{2}$ exceeds $D_{1}$ by $30 \%$ and more [38]. There are several potential explanations for the failure of the Weisskopf model in the present application. They mainly originate from insufficient knowledge of several cluster properties as discussed in detail in [38]. This failure is related to problems of decay rate theories in the explanation of the energy-dependence of observed decay rates as a function of excitation energy as discussed in [31].

Unfortunately, there is no sufficiently precise information on the monomer and dimer dissociation energies of the small, odd-size silver and copper cluster ions to quantitatively discuss the measured slopes as given in Table 1.

\section{Fragment Yields - Photoabsorption Cross Sections}

The observed total fragment yields can directly be converted to corresponding photodissociation cross sections. Under the present conditions of single-photon absorption which always leads to dissociation of the system and in the absence of other de-excitation channels as e.g., radiative cooling, the photodissociation cross section is identical to the photoabsorption cross section, since then the photodissociation yields are directly related to the optical properties of the cluster. These conditions are fulfilled, as justified above. The fraction of clusters that absorb a photon and thus undergo dissociation is given by [39] 


$$
\frac{n_{1}}{n_{0}}=\frac{1}{\beta} \int_{0}^{\infty} \exp \left(-\frac{4}{d_{C}^{2}} r^{2}\right)\left(1-e^{-\phi(r) \sigma}\right) d r
$$

where $\phi$ is the radial photon number density

$$
\phi(r)=\frac{4 \phi_{0}}{\pi d_{L}^{2}} \cdot \exp \left(-\frac{4}{d_{L}^{2}} r^{2}\right) .
$$

$\sigma$ is the absorption cross section for a single photon and $\beta$ is a dimensionless parameter that describes the overlap of the radial laser intensity distribution with the cluster density distribution. Both have been observed to be Gaussian-shaped. The parameter $\beta$ is defined as $d_{C}{ }^{2} / d_{L}{ }^{2}$ where $d_{C}$ is the cluster distribution diameter as determined earlier under similar experimental conditions [40] and $d_{L}$ is the laser beam diameter as defined above and as monitored by the CCD camera. For the present measurements, $\beta$ can be assumed to be close to unity, since $d_{L}$ is chosen to match $d_{C}$. However, it represents a source for systematic uncertainties.

The number of clusters which absorb two or more photons relative to the number of clusters which absorb one photon is for the present low laser intensities given by [39]

$$
\frac{n_{2}}{n_{1}} \approx \frac{1}{3} \frac{n_{1}}{n_{0}} .
$$

For most systems under investigation, relation (5) represents a suppression of higher-order photon absorptions with respect to single-photon absorption by a factor of more than 20. The only exceptions are the clusters $A g_{10}^{+}, A g_{12}^{+}, A g_{14}^{+}$and $A g_{16}^{+}$, for which the factor is only about 10 . But since dimer evaporation is no relevant decay channel for these systems anyway [35], all present results can be considered unperturbed by higher-order photoabsorption.

Thus, eq 3 can be numerically inverted to give the photoabsorption cross section $\sigma$ as a function of the measured fragment yield $n_{1} / n_{0}$. Figure 6 shows the measured fragment yields $n_{1} / n_{0}$ (left-hand scale) and the resulting photoabsorption cross sections from eq 3 (right-hand scale) which are also listed in Table 2.

The points marked with an arrow represent the cases in which the decay time constant is expected to be on the order of the experimental time window and thus both the fragment yield and the resulting cross section could be underestimated. In these cases, the present values can be considered a lower limit on the true values. For the points where the measured fragment yield is consistent with zero, the dissociation energy is known $[6,31,35]$ to exceed the excitation energy, so that no fragmentation can take place. In these cases, it is not possible to make a statement on the corresponding

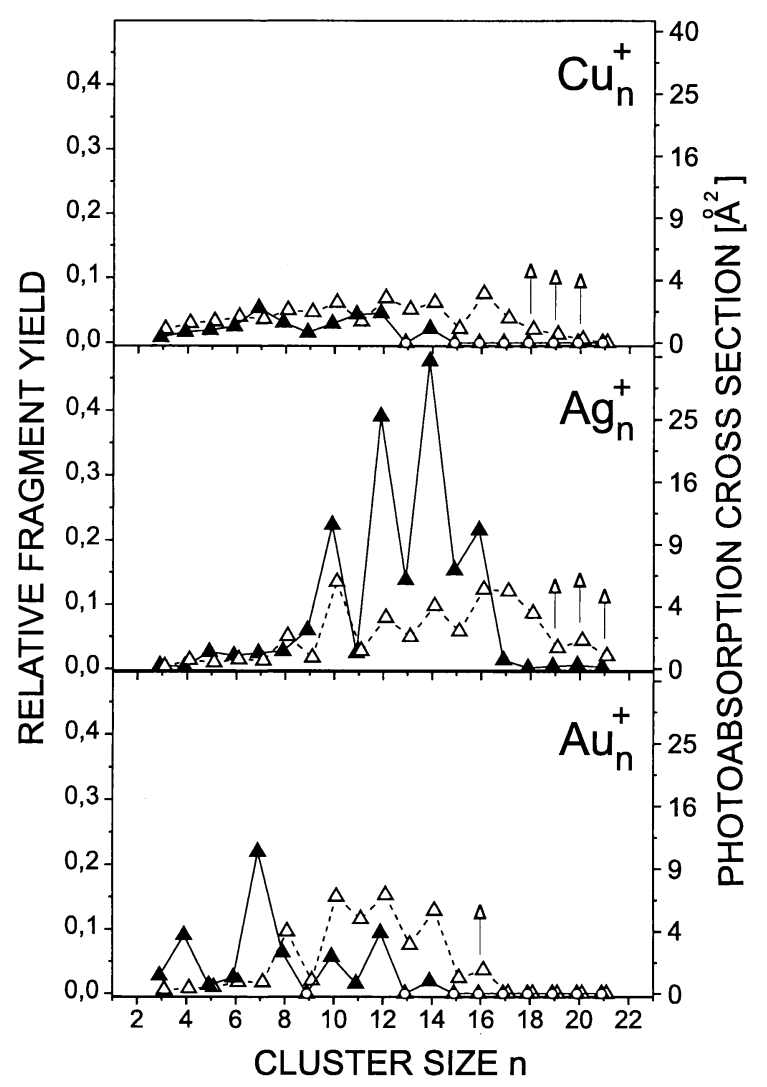

Figure 6. Observed fragment yield and corresponding photoabsorption cross section as a function of cluster size for clusters $\mathrm{Cu}_{n}{ }^{+}, \mathrm{Ag}_{n}{ }^{+}$and $\mathrm{Au}_{n}{ }^{+}(\mathrm{n}=3-21)$. Full triangles represent measurements at $\lambda=355 \mathrm{~nm}$, open triangles measurements at $\lambda=266 \mathrm{~nm}$. The error bars of the yields are smaller than the size of the symbols. Fragment yields identical with zero are marked with an open circle inside the triangle.

photoabsorption cross section. They are marked in Figure 6 with an open circle inside the triangle.

For better comparability, the frames in Figure 6 are plotted with a common scale. The fragment yields and thus photoabsorption cross sections are generally smallest for copper and largest for silver clusters. In all cases (both with respect to the different elements and photon energies) there is an odd-even effect as a function of cluster size. In the case of copper, generally the fragment yield for $\mathrm{E}=4.66 \mathrm{eV}$ dominates the yield for $\mathrm{E}=$ $3.49 \mathrm{eV}$. For silver, the situation is reversed. Gold clusters show a transition from dominance for $\mathrm{E}=3.49$ $\mathrm{eV}$ at small cluster sizes to dominance for $\mathrm{E}=4.66 \mathrm{eV}$ at larger sizes. The transition occurs between cluster sizes $\mathrm{n}=7$ and $\mathrm{n}=8$ and thus coincides with the transition from planar to 3-D structure [41, 42]. From the present measurements, however, it cannot be deduced that both effects are causally related. It is interesting to note that while the bulk behavior is quite similar for the three metals $[16,17]$, the size-dependence is obviously rather different in the case of small clusters. This effect may be attributed to the different dependence of electronic and geometric structures of the clusters as a function of the cluster size $n$, but no simple systematics was found. 
Table 2. Photoabsorption cross sections of noble metal cluster ions $C u_{n}^{+}, A g_{n}^{+}$and $A u_{n}^{+}(\mathrm{n}=3-21)$ in $\AA^{2}$. In every column the first number is the value measured at $\lambda=355 \mathrm{~nm}$ followed by a semicolon and the value measured at $\lambda=266 \mathrm{~nm}$.

\begin{tabular}{lccc}
\hline Size & $C u_{n}^{+}$ & $A g_{n}^{+}$ & $A u_{n}^{+}$ \\
\hline \hline 3 & $0.71(06) ; 0.97(16)$ & $0.66(04) ; 0.63(02)$ & $1.13(21) ; 0.67(04)$ \\
4 & $0.87(12) ; 1.17(23)$ & $0.62(02) ; 0.81(10)$ & $3.12(71) ; 0.71(06)$ \\
5 & $0.93(15) ; 1.25(24)$ & $1.09(20) ; 0.74(07)$ & $0.81(10) ; 0.74(07)$ \\
6 & $1.06(19) ; 1.42(31)$ & $0.96(16) ; 0.83(11)$ & $1.06(19) ; 0.91(14)$ \\
7 & $1.82(42) ; 1.34(28)$ & $1.05(19) ; 0.60(10)$ & $10.4(1.7) ; 0.70(08)$ \\
8 & $1.20(24) ; 1.71(39)$ & $1.12(21) ; 1.73(39)$ & $2.16(50) ; 3.86(87)$ \\
9 & $0.85(12) ; 1.63(37)$ & $2.40(52) ; 0.52(10)$ & $-; 0.78(08)$ \\
10 & $1.15(22) ; 2.07(48)$ & $10.7(1.7) ; 5.13(1.0)$ & $1.93(45) ; 5.95(1.1)$ \\
11 & $1.53(34) ; 1.25(25)$ & $1.04(19) ; 1.12(21)$ & $0.86(12) ; 4.05(88)$ \\
12 & $1.58(35) ; 2.32(54)$ & $26.7(3.1) ; 2.69(62)$ & $3.25(73) ; 6.06(1.2)$ \\
13 & $-; 1.75(40)$ & $5.31(1.0) ; 1.69(37)$ & $-; 3.64(85)$ \\
14 & $0.99(17) ; 2.08(48)$ & $37.6(3.7) ; 3.40(76)$ & $0.93(15) ; 4.51(1.0)$ \\
15 & $-; 0.99(17)$ & $6.13(1.2) ; 1.95(45)$ & $-; 0.72(08)$ \\
16 & $-; 2.54(59)$ & $10.1(1.6) ; 4.57(97)$ & $-; 1.88(34)$ \\
17 & $-; 1.37(29)$ & $0.82(10) ; 4.43(95)$ & $-;-$ \\
18 & $-; 0.95(15)$ & $0.59(01) ; 2.95(68)$ & $-;-$ \\
19 & $-; 0.80(10)$ & $0.63(03) ; 1.23(25)$ & $-;-$ \\
20 & $-; 0.65(04)$ & $0.66(04) ; 1.54(34)$ & $-;-$ \\
21 & $-;-$ & $0.61(10) ; 0.95(15)$ & $-;-$ \\
\hline
\end{tabular}

Table 3 gives an overview of experimental results on photoabsorption cross sections of noble metal cluster ions in those cases where previous results are available. In general, the present data agree with earlier results on gold and silver cluster ions produced under similar conditions [29, 43]. Systematic deviations are observed with respect to the cross sections of silver clusters reported by Tiggesbäumker et al. [44] which are larger than the present values. This may be due to the much higher temperature of the clusters well above $1000 \mathrm{~K}$ in the earlier investigation as compared to the clusters at room temperature in the present study. Photoabsorption cross section of metal clusters have earlier been observed to increase with rising cluster temperature [45, 17]. Deviations are also found with respect to the cross

Table 3. Comparison of values of photoabsorption cross sections of noble metal cluster ions where other experimental results are available

\begin{tabular}{lccll}
\hline System & $\mathrm{E}[\mathrm{eV}]$ & $\begin{array}{c}\sigma(E)\left[\AA^{2}\right] \\
\text { this work }\end{array}$ & $\begin{array}{c}\sigma(E)\left[\AA^{2}\right] \\
\text { other work }\end{array}$ & Ref. \\
\hline \hline$A g_{9}^{+}$ & 3.49 & $2.40(52)$ & $2.7(7)$ & {$[44]$} \\
$A g_{9}^{+}$ & 3.49 & $2.40(52)$ & $1.2(2)$ & {$[43]$} \\
$A g_{9}^{+}$ & 4.66 & $0.52(10)$ & $0.8(2)$ & {$[44]$} \\
$A g_{11}^{+}$ & 3.49 & $1.04(19)$ & $4.1(1.2)$ & {$[44]$} \\
$A g_{11}^{+}$ & 4.66 & $1.12(21)$ & $1.6(4)$ & {$[44]$} \\
$A g_{15}^{+}$ & 3.49 & $6.13(1.2)$ & $7.2(1.6)$ & {$[44]$} \\
$A g_{15}^{+}$ & 4.66 & $1.59(45)$ & $2.4(6)$ & {$[44]$} \\
$A g_{21}^{+}$ & 3.49 & $0.61(10)$ & $9.1(1.8)$ & {$[44]$} \\
$A g_{21}^{+}$ & 4.66 & $0.95(15)$ & $1.1(3)$ & {$[44]$} \\
$A u_{9}^{+}$ & 4.66 & $0.78(08)$ & $0.4(1)$ & {$[43]$} \\
$A u_{11}^{+}$ & 3.49 & $0.86(12)$ & 0.45 & {$[46]$} \\
$A u_{11}^{+}$ & 4.66 & $4.05(88)$ & 0.4 & {$[46]$} \\
$A u_{13}^{+}$ & 3.49 & - & 0.35 & {$[46]$} \\
$A u_{13}^{+}$ & 4.66 & $3.64(85)$ & 0.3 & {$[46]$} \\
$A u_{15}^{+}$ & 4.66 & $0.72(08)$ & $0.36(4)$ & {$[29]$} \\
$A u_{21}^{+}$ & 4.66 & - & $0.4(2)$ & {$[43]$} \\
\hline
\end{tabular}

sections reported by Collings [46]. These values result from measurements on gold cluster ions with an attached Xe atom, which thus seems to change the properties of the system significantly.

\section{Conclusion}

Stored size-selected cluster ions $C u_{n}^{+}, A g_{n}^{+}$and $A u_{n}^{+}$ ( $n=3-21)$ have been photodissociated with 10-ns laser pulses of low intensity at wavelengths of $355 \mathrm{~nm}$ and $266 \mathrm{~nm}$ under well-defined and constant conditions for a direct comparison between the different species. The decay pathway branching ratios between neutral monomer and neutral dimer evaporation in unimolecular single-photon dissociation have been measured as a function of cluster size, excitation energy and element. Even-size cluster ions show exclusive monomer evaporation irrespective of their size. In contrast, for odd-size cluster ions there is a competition by dimer evaporation. The relative dimer fragment yield shows a characteristic transition from dimer evaporation in the case of small clusters to monomer evaporation in the case of larger clusters. Nine-atomic cluster ions represent an exemption from the transition: they show a significantly smaller dimer fragment yield than their odd-size neighbors with 7 and 11 atoms. The slopes of the dependence of the decay pathway branching ratio on the excitation energy have been determined. In the case of silver, the measured slopes are positive as expected from decay rate theory. In contrast, the slopes are negative for copper and gold clusters. In the case of gold, the experimental results disagree with expectations according to the Weisskopf formalism. However, this disagreement is in line with earlier findings [31].

Furthermore, the total fragment yields have been monitored as a function of cluster size. Besides a 
pronounced odd-even effect, the fragment yields show a significant dependence on both the wavelength and the element under investigation. Under the present experimental conditions, the observed fragment yields can be converted to the corresponding photoabsorption cross sections. In those few cases, where earlier data are available, in general the results agree. Deviations are noted in the case of silver and of gold due to temperature effects and adsorbant effects, respectively.

\section{Acknowledgments}

This work was funded by the Deutsche Forschungsgemeinschaft and the EU network "CLUSTER COOLING." The authors further thank the Fonds der Chemischen Industrie for financial support.

\section{References}

1. Bréchignac, C.; Cahuzac, Ph.; Leygnier, J.; Weiner, J.; et al. Dynamics of Unimolecular Dissociation of Sodium Cluster Ions. J. Chem. Phys. 1989, 90, 1492.

2. Bréchignac, C.; Cahuzac, Ph.; Carlier, F.; et al. Cohesive Energies of $K_{n}^{+} 5<n<200$ from Photoevaporation Experiments. J. Chem. Phys. 1990, 93, 7449.

3. Bréchignac, C.; Busch, H.; Cahuzac, Ph.; et al. Dissociation Pathways and Binding Energies of Lithium Clusters from Evaporation Experiments. J. Chem. Phys. 1994, 101, 6992.

4. Becker, S.; Dietrich, G.; Hasse, H.; et al. Collision Induced Dissociation of Stored Gold Clusters Ions. Z. Phys. D. 1994, 30, 341.

5. Krückeberg, S.; Dietrich, G.; Lützenkirchen, K.; et al. The Dissociation Channels of Silver Clusters $\mathrm{Ag}_{n}{ }^{+}, 3 \leq n \leq 20$. Int. J. Mass. Spectrom. Ion Proc. 1996, 155, 141.

6. Hild, U.; Dietrich, G.; Krückeberg, S.; et al. Time Resolved Photofragmentation of Stored Silver Clusters $\mathrm{Ag}_{n}{ }^{+}$(n $=8$ to 21). Phys. Rev. A 1998, 57, 2786.

7. Weidele, H.; Vogel, M.; Herlert A.; et al. Decay Pathways of Stored Metal Cluster Anions after Collisional Activation. Eur. Phys. J. D. 1999, 9, 173.

8. Spasov, V. A.; Lee, T. H.; Maberry, J. P.; et al. Measurement of the Dissociation Energies of Anionic Silver Clusters $\mathrm{Ag}_{n}^{-}, \mathrm{n}=$ 2-11 by Collision-Induced Dissociation. J. Chem. Phys. 1999, $110,5208$.

9. Ingolfsson, O.; Busolt, O.; Sugawara, K.; et al. Energy-Resolved Collision-Induced Dissociation of $\mathrm{Cu}_{n}^{+}(\mathrm{n}=2-9)$ : Stability and Fragmentation Pathways. J. Chem. Phys. 2000, 112, 4613.

10. Spasov, V. A.; Lee, T. H.; Ervin, K. M. Threshold CollisionInduced Dissociation of Anionic Copper Clusters and Copper Cluster Monocarbonyls. J. Chem. Phys. 2000, 112, 1713.

11. Spasov, V. A.; Shi, Y.; Ervin, K. M.; et al. Time-Resolved Photoabsorption and Threshold Collision-Induced Dissociation of Anionic Gold Clusters. Chem. Phys. 2000, 262, 75.

12. Krückeberg, S.; Schweikhard, L.; Ziegler, J.; et al. Decay Pathways and Dissociation Energies of Copper Clusters, $\mathrm{Cu}_{n}^{+}$ $(2 \leq n \leq 25), \mathrm{Cu}_{n}^{2+}(15 \leq n \leq 25)$. J. Chem. Phys. 2001, 114, 2955.

13. Vogel, M.; Hansen, K.; Herlert, A.; Schweikhard, L. Decay Pathways of Small Gold Clusters. Eur. Phys. J. D. 2001, 16, 73.

14. Vogel, M.; Hansen, K.; Herlert, A.; Schweikhard, L. Energy Dependence of the Decay Pathway of Optically Excited Gold Clusters. Appl. Phys. B. 2001, 73, 411.

15. Hansen, K. Statistical Emission Process of Clusters. Philos. Mag. B. 1999, 79, 1413.
16. Weaver, J. H.; Frederikse, H. P. R. CRC Handbook, 78th ed., D.R. Lide, Ed. CRC Press: Boca Raton, FL, 1998.

17. Kreibig, U.; Vollmer, M. Optical Properties of Metal Clusters. Springer Series in Materials Science, Vol. 25. Springer-Verlag: New York, NY; Berlin, Germany, 1995.

18. Quinten, M. Optical Constants of Gold and Silver Clusters in the Spectral Range Between 1.5 and 4.5 eV. Z. Phys. B. 1996, 101, 211.

19. Lindinger, M.; Dasgupta, K.; Dietrich, G.; et al. Time Resolved Photofragmentation of $\mathrm{Au}_{n}{ }^{+}$and $\mathrm{Ag}_{n}{ }^{+}$Clusters. Z. Phys. D. 1997, 40, 347.

20. Schweikhard, L.; Becker, S.; Dasgupta, K.; et al. Trapped Metal Cluster Ions. Physica Scripta 1995, T59, 236.

21. Becker, S.; Dasgupta, K.; Dietrich, G.; et al. A Penning Trap Mass Spectrometer for the Study of Cluster Ions. Rev. Sci. Instrum. 1995, 66, 4902.

22. Schweikhard, L.; Krückeberg, S.; Lützenkirchen, K.; et al. The Mainz Cluster Trap. Eur. Phys. J. D. 1999, 9, 15.

23. Dietz, T. G.; Duncan, M. A.; Powers, D. E.; Smalley, R. E. Laser Production of Supersonic Metal Cluster Beams. J. Phys. Chem. 1981, 74, 6511.

24. Weidele, H.; Frenzel, U.; Leisner, T.; Kreisle, D. Production of Cold/Hot Metal Cluster ions: a modified laser vaporization source. Z. Phys. D. 1991, 20, 411.

25. Brown, L. S.; Gabrielse, G. Physics of an Electron or Ion in a Penning Trap. Rev. Mod. Phys. 1986, 58, 233.

26. Schnatz, H.; Bollen, G.; Dabkievicz, P.; et al. In-Flight Capture of Ions into a Penning Trap. Nucl. Instrum. Meth. 1986, A251, 17.

27. Savard, G.; Becker, S.; Bollen, G.; et al. A New Cooling Technique for Heavy Ions in a Penning Trap. Phys. Lett. A 1991, 158, 247.

28. Guan, S. H.; Marshall, A.; et al. Shrink-Wrapping an Ion Cloud for High-Performance Fourier-Transform Ion-Cyclotron Resonance Mass Spectrometry. Chem. Rev. 1994, 94, 2161.

29. Walther, C.; Dietrich, G.; Lindinger, M.; et al. Time-Resolved Photofragmentation of $\mathrm{Au}_{15}^{+}$. Chem. Phys. Lett. 1996, 256, 77 and (1996), 262, 668.

30. Schweikhard, L.; Dietrich, G.; Hild, U.; et al. Dissociation Studies of Metal Clusters. Rapid Commun. Mass Spectrom. 1997, 11,1624 .

31. Vogel, M.; Hansen, K.; Herlert, A.; Schweikhard, L. ModelFree Determination of Dissociation Energies of Polyatomic Systems. Phys. Rev. Lett. 2001, 87, 013401.

32. Walther, C.; Dietrich, G.; Dostal, W.; et al. Radiative Cooling of a Small Metal Cluster: The Case of $\mathrm{V}_{13}^{+}$. Phys. Rev. Lett. 1999, 83, 3816.

33. Katakuse, I.; Ichihara, T.; Fujita, Y.; et al. Mass Distributions of Copper, Silver and Gold Clusters and Electronic Shell Structure. Int. J. Mass. Spectrom. Ion Proc. 1985, 67, 229.

34. deHeer, W. A. The Physics of Simple Metal Clusters: Experimental Aspects and Simple Models. Rev. Mod. Phys. 1993, 65, 611.

35. Krückeberg, S.; Dietrich, G.; Lützenkirchen, K.; et al. MultipleCollision Induced Dissociation of Trapped Silver Clusters $\mathrm{Ag}_{n}^{+}, 2 \leq n \leq 25$. J. Chem. Phys. 1999, 110, 7216 .

36. Weisskopf, V. Statistics and Nuclear Reactions. Phys. Rev. 1937, 52, 295.

37. Frauendorf, S. Evaporation Rates for Liquid Clusters. Z. Phys. D. 1995, 35, 191.

38. Vogel, M.; Hansen, K.; Herlert, A.; Schweikhard, L. Dimer Dissociation Energies of Small Odd-Size Clusters $\mathrm{Au} u_{n}^{+}$. Eur. Phys. J. D. 2002, 21, 163.

39. Walther, C.; Becker, S.; Dietrich, G.; et al. Photo Fragmentation of Metal Clusters Stored in a Penning Trap. Z. Phys. D. 1996, 38,51 . 
40. Walther, C.; Dietrich, G.; Dostal, W.; et al. The Temperature Dependence of Photoabsorption of $\mathrm{V}_{13}^{+}$. Eur. Phys. J. D. 1999, 9, 455.

41. Rousseau, R.; Dietrich, G.; Krückeberg, S.; et al. Probing Cluster Structures with Sensor Molecules: Methanol Adsorbed Onto Gold Clusters. Chem. Phys. Lett. 1998, 295, 41.

42. Gilb, S.; Weis, P.; Furche, F.; et al. Structures of Small Gold Cluster Cations $\left(\mathrm{Au}_{n}^{+}, \mathrm{n}<14\right)$ : Ion Mobility Measurements Versus Density Functional Calculations. J. Chem. Phys. 2002, $116,4094$.

43. Lindinger, M.; Dasgupta, K.; Dietrich, G.; et al. Time Resolved
Photofragmentation of $\mathrm{Au}_{n}^{+}$and $\mathrm{Ag}_{n}^{+}$Clusters. Z. Phys. D. 1997, 40, 347

44. Tiggesbäumker, J.; Köller, L.; Lutz, H.; Meiwes-Broer, K. Giant Resonances in Silver-Cluster Photofragmentation. Chem. Phys. Lett. 1992, 190, 42.

45. Reiners, Th.; Orlik, W.; Ellert, Ch.; et al. Temperature Dependence of the $\mathrm{Na}_{n}^{+}$Optical Response. Chem. Phys. Lett. 1993, 215, 357.

46. Collings, B. A.; Athanassenas, K.; Lacombe, D.; et al. Optical Absorption Spectra of $A u_{7}, A u_{9}, A u_{11}$ and $A u_{13}$ and Their Cations. J. Chem. Phys. 1994, 101, 3506. 